\title{
Cancer Immunotherapy "HLA-G an Important Neglected Immunosuppressive Molecule"
}

\author{
Robert L. Elliott* \\ Elliott Mastology Center, Baton Rouge, LA 70806, USA
}

Received: June 10, 2016; Accepted: June 23, 2016; Published: June 30, 2016

*Corresponding author: Robert L Elliott, Elliott Mastology Center, Baton Rouge, LA. 70806, USA, E-mail: shamichelle2911@outlook.com

\begin{abstract}
Absract
Renewed interest in cancer immunotherapy has emerged by the introduction of targeted monoclonal antibodies and checkpoint blockade inhibitors. Adaptive cancer immunotherapy will be much more effective by a combination approach. A limiting factor is the multitude of tumor immunosuppressive factors. A neglected molecule is HLA-G antigen expression by malignant cells; which allows the escape of tumor cells from Natural Killer Cell (NK) and cytotoxic CD8+ T-cell destruction. Though much has been published about HLA-G as a cancer immunosuppressant molecule, little has been done to attack HLA-G as a cancer therapeutic target. In this review, we hope to stimulate researchers to investigate the role of HLA-G as a therapeutic target of cancer Immunosuppression.
\end{abstract}

\section{Introduction}

Human leukocyte antigen G (HLA-G) is an immunotolerant non-classical major histocompatibility complex $1 \mathrm{~b}$ molecule. It is normally expressed in placenta trophoblastic cells at the maternal-fetal interface [1]. HLA-G expression was later demonstrated in human tumors and was thought to play a role of Immunosuppression in the tumor microenvironment [2].

Paul, et. al. [3] was first to show evidence that HLA-G protein expression could play a role in tumor escape from the host immune system in 1998. They showed that melanoma cells were protected from NK cell mediated cytolysis by HLA-G expression. Carosella, et al. [4] has investigated the complexities of HLA-G as an immunomodulatory molecule, and its role in tumor immunosuppression.

Uroservic and Dammer [5] suggested that HLA-G was associated with tumor progression and growth, and that it was mainly detected in tumor tissue and rarely in adjacent normal tissue. They described in great detail the role of HLA-G in cancer immunoediting: (1) elimination, (2) equilibrium, and (3) escape were discussed in detail. We recently reported on HLA-G expression in breast cancer and its role in Immunosuppression [6]. Since that report more research has convinced us that HLA-G plays a major role of Immunosuppression in the tumor microenvironment. The purpose of this review is to bring more awareness of the role of HLA-G in cancer Immunosuppression, which in the present field of cancer immunotherapy, in my opinion has been severely neglected.

\section{Evidence HLA-G Plays a Significant Role in Tumor Immunosuppression}

Curigliano and Criscitiello, et al. [7] have addressed the molecular pathways of HLA-G. There are seven isoforms, four membrane bound (HLA-G1 to HLA-G-4); and three secreted isoforms (s HLA-G; HLA-G5 to HLA-G7). HLA-G5 is the soluble counterpart of HLA-G1; and most information known concerns the HLA-G1 molecule and its soluble counterpart HLA-G5. HLA-G induces tolerance and tumor Immunosuppression by inhibiting various immune-competent cells. The suppressive effect is caused by the binding of both soluble and membrane bound HLA-G to inhibitory receptors of effector immune cells, especially the Killer Cell Immunoglobulin-like Receptor (KIR) expressed on (NK) cells [7].

Others have reported that HLA-G antigens are expressed on tumor infiltrating cells and can be detected in the blood of cancer patients. While Le Maoult, et al. [8] reported that HLA-G has the capability to induce regulatory T-cells. CD4+ and CD8+ $\mathrm{T}$-cells lose their ability to respond to antigenic stimulation in the presence of HLA-G and differentiate into regulartory T-cells which inhibit other reactive T-cells. There are many more HLA-G immune functions that are beyond the scope of this review.

\section{More Research Evidence of HLA-G Tumor Immunosuppression}

Rouas-Freiss, et al. [9] have done a great job discussing HLA-G proteins in cancer and how they provide tumor cells with an immune escape mechanism. They report on the ability of HLA-G to suppress cellular immune functions. HLA-G inhibits (NK) cell and CTL-mediated cytolysis and T-cell proliferative response. HLA-G also exerts its Immunosuppression effect by allowing the cell surface expression of HLA-E through an indirect pathway, HLA-E inhibits NK and T-cells by interacting with their inhibitory receptors [2].

HLA-G has the ability to modulate and cause release of cytokines directing the tumor micro-environment to a Th2 profile. One such cytokine is IL-10, a powerful immunosuppressive cytokine, which confers immunoprotection to tumor cells and promotes metastases. HLA-G expression has been shown to be present in numerous cell type malignancies and increased levels 
of soluble HLA-G levels have been detected in serum and ascites of patients with cancer. The increased expression of HLA-G in cancer tissue and serum is a bad prognostic indicator. Patient's survival from glioblastoma multiforme after diagnosis is inversely correlated with their serum HLA-G level. This fact indicates that HLA-G expression in the tumor should be evaluated to determine the efficacy of the immunotherapy protocol of malignant disease $[10,11]$.

Ines Zidi and her associates have contributed tremendously to the role of HLA-G in tumorigenesis, cancer progression, metastasis, transplantation, autoimmunity and other diseases. She and Ben Amor have published an extensive study on HLA-G regulators in medicine and an outline of key requirements. They emphasize the importance of screening for HLA-G expression after cancer therapy and believe HLA-G could be implicated as a therapeutic target in cancer therapy. They propose that HLA-G is a key player in cancer Immunosuppression [12]. Increased HLA-G gene expression in primary cancer cells and cell lines is associated with immune suppression and worsens the prognosis of the cancer patient $[9,13]$. Downregulation of HLA-G gene expression aids in destroying cancer cells and improves patient outcome [13]. Zidi and Ben Amor discuss regulators of HLA-G gene expression both inhibitors and stimulators, such as enzymes, hormones, hormone antagonist, cytokines, radiation, (HDAC) inhibitors and the iron chelator desferrioxamine. They stress monitoring serum HLA-G levels and that clinical studies should concentrate on the importance of HLA-G gene expression during cancer therapy [12].

\section{HLA-G Promotes Metastasis}

Zidi and Ben Amor [13] have reported on HLA-G as a promoter of metastasis and that its powerful immune functions could have a pivotal role in metastatic progression. Trogocytosis and hypoxia can probably transform HLA-G negative tumor cells into positive ones: and cause tumor progression. HLA-G binding to epithelium integrins with great avidity enhances extravasation and migration of tumor cells. Evidence shows that HLA-G in soluble forms in plasma and bound tumor tissue forms is associated with poor patient out-come. They state this molecule may represent a valuable target in the design of future therapies for invasive and metastatic cancer [14]. Michelin S, et al. [15] has reported on HLA-G as a target molecule in specific immunotherapy against renal cell carcinoma. They discuss many mechanisms of HLA-G peptide vaccination techniques. Their work supports the fact that HLA-G should be evaluated and attacked therapeutically in malignancy especially renal cell carcinoma.

\section{HLA-G Expression, Molecular Pathways and Effect of Systemic Treatment}

Curigliano and Criscitiello, et al. [7] have reported on HLA-G and its molecular pathways to induce tumor Immunosuppression. They describe that the ectopic expression of HLA-G is observed in cancer. It is definitely a strategy used by tumor cells to escape immune surveillance. They focus on the association of HLA-G expression in cancer and patient prognosis. They define molecular mechanisms of defective HLA-G expression, function of HLA-G immune tolerant tumors, potential diagnostic use of soluble and membrane HLA-G as a biomarker to indentify and monitor stage of disease. It is pointed out that the blockade of HLA-G is a very attractive therapeutic technique against cancer [7].

Important therapies for cancer, such as, radiation, chemotherapy, immunotherapy and biologic agents can be paradoxical and induce HLA-G expression. The possible increase in HLA-G production by therapy could affect therapeutic response. Therefore, measurement of HLA-G is desired [16]. There are three important mechanisms of immune escape as follows: (1) cytotoxic cell inhibition, (2) induction of suppressor $\mathrm{T}$-cells, and (3) interference of antigen presentation by dendritic cells. All of these and high HLA-G levels promote metastasis and a poor prognosis [17].

\section{HLA-G Gene Expression in Tumor Cells "The Role Of Hypoxia"}

Zidi and her collaborators [13] have done a great job researching the role of hypoxia in the modulation of HLA-G gene expression in tumor cells. They state the regulatory mechanisms of HLA-G expression differ from classical HLA class I genes. There is a tight control of HLA-G gene expression in tumor cells by cis-acting epigenetic mechanisms. They believed that those processes were dependent on microenvironment conditions, particularly stress conditions like hypoxia. Cellular response to hypoxia is driven by the transcription factor, hypoxia inducible factor 1 (HIF-1). They showed that HLA-G gene expression in hypoxic tumors is dependent on (HIF-1) stabilization. The processes of HLA-G expression are dependent on tumor microenvironment conditions particularly stress. Zidi's group and Zhong and De Marzo, et al. [18] have concluded that hypoxia is related to tumor stress that could induce (HIF-1) dependent HLA-G transcription in tumor cells. The overexpression of (HIF1) being seen in human cancers and metastatic disease. HLA-G expression on the surface of tumor cells exposed to hypoxia promotes tumor Immunosuppression and favors tumor growth.

Forte, Pazmany, Matter-Reissmann, et al. [19] have shown that HLA-G inhibits the rolling adhesion of activated human NK cells on porcine endothelial cells. HLA-G may also be expressed in the abnormal vasculature of the tumor microenvironment; thus preventing roll, sticking and extravasation of $\mathrm{NK}$ and CD8+ cytotoxic lymphocytes from entering the tumor microenvironment and attacking the cytolysis and destruction of tumor cells. I am and have been convinced that an important factor in the tumor microenvironment is dysfunction of adhesion molecules in the tumor vasculature. HLA-G might just be the culprit that initiates that process.

\section{Conclusion}

\section{HLA-G a neglected therapeutic target}

We have presented much research evidence that in epithelial cancers HLA-G should be considered a therapeutic target. This is especially true if the patient has metastatic disease, because HLA-G expression will impact the treatment protocol response. 
This includes response to chemotherapy, radiation, biotherapy and immunotherapy protocols. As a surgical oncologist, I participate in cancer meetings of all of those modalities and never hear anything about HLA-G. This is even true of the cancer immunotherapy meetings, where it should be a major topic. Serum and tissue from Stage IV patients should be checked for HLA-G. It can provide information about tumor burden and response to treatment. Attacking and blocking HLA-G will improve the response of cancer immunotherapy, rather passive, adoptive, adaptive, and even check point blockade.

There are antibodies directed to HLA-G but I know of none used in clinical cancer immunotherapy protocols. This is a must because for cancer immunotherapy to be most effective there has to be a combination approach. Everything must be done to inhibit tumor Immunosuppression, stimulate the effector system, and allow NK cells and cytotoxic CD8+ T-cells to enter the tumor microenvironment. Blocking HLA-G might be the first step, as I believe HLA-G is the Father of tumor Immunosuppression that initiates the immunosuppressive cascade. There is no doubt that more attention should be paid to this very important molecule involved in cancer immune evasion and progression. HLA-G should be considered as a target in most cancer immunotherapeutic protocols, and that time is NOW!

\section{References}

1. Kovats S, Main EK, Librach C, Stubblebine M, Fisher SJ, DeMars R, etal. A Class I antigen, HLA-G, expressed in human trophoblast. Science. 1990;248(4952):220-3.

2. Rouas-Freiss N, Moreau P, Ferrone S, Carosella ED. HLA-G proteins in cancer: Do they provide tumor cells with an escape mechanisms? Cancer Res. 2005;65(22):10139-44.

3. Paul P, Rouas-Freiss N, Khalil-Daher I etal, HLA-G expression in melanoma: A way for tumor cells to escape from immunosurveillance. Proc Natl Acad Sci U S A. 1998;95(8):4510-5.

4. Carosella ED, Favier B, Rouas-Freiss N, Moreau P, Lemaoult J. Beyond the increasing complexity of the immunomodulatory HLA-G molecule. Blood. 2008;111(10):4862-70. doi: 10.1182/blood-2007-12-127662.

5. Urosevic M, Dummer R. Human leukocyte antigen-G and cancer immunoediting. Cancer Res. 2008;68(3):627-30. doi: 10.1158/00085472.CAN-07-2704.

6. Elliott RL, Jiang XP, Phillips JT, Barnett BG, Head JF. Human leukocyte antigen $\mathrm{G}$ expression in breast cancer; Role in Immunosuppression. Cancer Biother Radiopharm. 2011;26(2):153-7. doi: 10.1089/ cbr.2010.0924.
7. Curigliano G, Criscitiello C, Gelao L, Goldhirsch A. Molecular Pathways: Human leukocyte antigen G (HLA-G). Clin Cancer Res. 2013;19(20):5564-71. doi: 10.1158/1078-0432.CCR-12-3697.

8. Le Maoult J, Krawice-Radanne I, Dousset J, Carosella ED. HLA-G1 expressing antigen-presenting cells induce immuno-suppressive CD4+ T-cells. Proc Natl Acad Sci U S A. 2004;101(18):7064-9.

9. Rouas-Freiss N, Moreau P, Menier C, Carosella ED. HLA-G in cancer: a way to turn off the immune system. Semin Cancer Biol. 2003;13(5):325-326.

10. Schütt P, Schütt B, Switala M, Bauer S, Stamatis G, Opalka B, et al. Prognostic relevance of soluble human leukocyte antigenclass 1 molecules in lung cancer patients. Hum Immunol. 2010;71(5):48995. doi: 10.1016/j.humimm.2010.02.015.

11.Zidi I, Ben Amor N. HLA-G regulators in cancer medicine: an outline of key requirements. Tumour Biol. 2011;32(6):1071-86. doi: 10.1007/s13277-011-0213-2.

12. Cabestre FA, Lefebvre S, Moreau P, Rouas-Friess N, Dausset J, Carosella ED, et al. HLA-G expression: immune privilege for tumor cells? Semin Cancer Biol. 1999;9(1):27-36.

13.Zidi I, Ben Amor N. HLA-G as predisposing for metastasis. Med Hypotheses. 2011;77(1):134-9. doi: 10.1016/j.mehy.2011.03.046.

14. Komohara Y, Harada M, Ishihara Y, Suekane S, Noguchi M, Yamada A, et al. HLA-G as a target molecule in specific immunotherapy against renal cell carcinoma. Oncology Reports. 2007;18:1463-1468.

15. Michelin S, Gallegos CE, Dubner D, Favier B, Carosella ED. Ionizing radiation modulates the surgace expression of human leukocyte antigen-G in a human melanoma cell line. Hum Immunol. 2009;70(12):1010-5. doi: 10.1016/j. humimm.2009.07.030.

16. Dunn GP, Bruce AT, Ikeda H, Old LJ, Schreiber RD. Cancer immunoediting: from immunosurveillance to tumor escape. Nat Immunol. 2002;3(11):991-8.

17. Mouillot G, Marcou C, Zidi I, Guillard C, Sangrouber D, Carosella ED, et al. Hypoxia modulates HLA-G Gene Expression in Tumor Cells. Hum Immunol. 2007;68(4):277-85.

18. Zhong H, De Marzo AM, Laughner E, Lim M, Hilton DA, Zagzag D, et al. Overexpression of hypoxica in ducible factor 1 alpha in common human cancers and their metastases. Cancer Res. 1999;59:5830.

19. Forte P, Pazmany L, Matter-Reissmann UB, Stussi G, Schneider MK, Seebach JD. HLA-G Inhibits rolling adhesion of activated human NK cells on porcine endothelial cells. J Immunol. 2001;167(10):6002-8. 